The Canadian Journal of Higher Education, Vol. XXV-3, 1995

La revue canadienne d'enseignement supérieur, Vol. XXV-3, 1995

\title{
L'évolution du contrat social de la recherche universitaire: contraintes et opportunités
}

\author{
RÉJEAN LANDRY*
}

\section{Résumé}

Les mutations en cours dans le financement de la recherche universitaire contribuent à intensifier la réflexion sur l'interdépendance croissante qui existe entre les universités et le gouvernement. Au fil du temps, ces liens d'interdépendance ont fini par faire émerger une sorte de contrat social, d'abord implicite, puis progressivement, de plus en plus explicite, contenant des clauses de plus en plus nombreuses et de plus en plus détaillées pour régir les liens d'interdépendance qui existent entre les universités et le gouvernement. L'article démontre que les clauses de ce contrat social ont été révisées progressivement sous la pression de changements graduels qui sont survenus tant du côté de la recherche universitaire que du côté gouvernemental. L'examen. de ces changements amène l'auteur à conclure que le contrat social qui lie les universités au gouvernement sera de plus en plus contraignant. La dernière partie de l'article propose de considérer ces contraintes de façon positive en tentant de voir les opportunités qui s'en dégagent pour l'avenir du financement de la recherche universitaire en suggérant un certain nombre d'actions concrètes s'adressant tant aux chercheurs qu'aux gestionnaires des universités.

\section{Abstract}

Ongoing mutations in the funding of university research lead to the intensification of the reflexions on the increasing interdependence linking the universities to the governments. Through the years, these linkages of

* Université Laval 
interdependence have generated a sort of social contract that was initially implicit, but has become increasingly more and more explicit, including increasing number of provisions governing the linkages prevailing between the universities and the governments. The paper demonstrates that the provisions of this social contract have been gradually revised under the pressures of changes that gradually occurred concerning the views of the government policy makers and the needs and practices of university research. The examination of these changes incites the author to conclude that the social contract relating the universities to the governments will become more and more compelling. The last part of the paper proposes to consider these constraints in a positive manner in attempting to figure out opportunities opened for future funding of university research. Specific lines of lobbying actions are suggested to improve the marketing of university research.

Les mutations en cours dans le financement de la recherche universitaire contribuent à intensifier la réflexion sur l'interdépendance croissante qui existe entre les universités et le gouvernement. Au fil du temps, ces liens d'interdépendance ont fini par faire émerger une sorte de contrat social, d'abord implicite, puis progressivement, de plus en plus explicite, contenant des clauses de plus en plus nombreuses et de plus en plus détaillées pour régir les liens d'interdépendance qui existent entre les universités et le gouvernement. Je tenterai de démontrer que les clauses de ce contrat social ont été révisées, non pas de façon abrupte, mais progressivement, sous la pression de changements graduels qui sont survenus tant du côté de la recherche universitaire que du côté gouvernemental. L'examen de ces changements m'amènera à conclure que le contrat social qui lie les universités au gouvernement sera de plus en plus contraignant. Finalement, je proposerai de considérer ces contraintes de façon positive en tentant de voir les opportunités qui s'en dégagent pour l'avenir du financement de la recherche universitaire en suggérant un certain nombre d'actions concrètes s'adressant tant aux chercheurs qu'aux gestionnaires des universités. Mais d'abord, quelle réalité interpelle-t-on avec cette idée de contrat?

\section{Le contrat social de la recherche}

Guston et Keniston, (1994) suggèrent de considérer la relation qui existe entre les universités et le gouvernement en matière de recherche sous l'angle d'un contrat où, d'une part, le gouvernement s'engage à subventionner les projets qui, suivant les critères d'excellence utilisés par les pairs, contribuent à l'avancement des connaissances alors que, d'autre part, les chercheurs universitaires s'engagent à utiliser les fonds gouvernementaux pour produire 
des connaissances qui débouchent sur le développement de nouveaux produits, de nouveaux médicaments, de nouvelles solutions pour résoudre les problèmes socio-économiques. On peut parler d'un contrat parce qu'on assiste à un échange de ressources et de services entre le gouvernement et les universités. Il importe toutefois de souligner qu'il s'agit d'un échange de ressources pour des services qui ne sont pas encore produits et dont la quantité et la qualité sont déterminées par les décisions à prendre par les chercheurs au fur et à mesure qu'ils réalisent leurs projets. En ce sens, le contrat social de la recherche correspond à un pari sur le futur.

Le contrat social de la recherche vise donc à la fois à fournir un instrument de gestion de l'échange ainsi qu'un instrument de production de résultats. Comme toute entente contractuelle, le contrat social de la recherche a pour objectif de coordonner les décisions des contractants, garantir la réalisation de leurs promesses et, finalement, répartir les résultats issus de la coopération entre les contractants (Brousseau, 1993, p. 111). En pratique, un contrat constitue la somme des clauses organisant la coordination, le système de garantie et de surveillance, les règles de partage des résultats ainsi que la durée des engagements. L'élaboration des clauses qui définissent les arrangements contractuels dépendent des caractéristiques des transactions ainsi que de l'efficacité des différentes clauses qui organisent les transactions. Au fil du temps, les parties prenantes à un contrat sont incités à renégocier les clauses contractuelles qui tendent à devenir moins efficaces.

Avant d'aller plus loin, il importe de caractériser les transactions susceptibles d'être conclues dans le cadre du contrat social de la recherche. Des travaux sur la théorie économique des contrats, je retiendrai que le succès des transactions dépend de trois traits génériques (Brousseau, 1993; Bryson \& Smith Ring, 1990; Williamson, 1985):

- le degré de spécificité des actifs engagés dans une transaction se définit en termes de redéployabilité: suivant Williamson (1985, pp. 66-84) un actif indifférencié renvoie à une ressource peu spécialisé, disponible en abondance et facilement redéployable pour d'autres d'usages et d'autres transactions alors qu'à l'inverse un actif idiosyncratique est très spécialisé, rare et difficilement redéployable.

- le degré d'incertitude renvoie aux changements qui surviennent tant du côté de la quantité et de la qualité de ce qui est échangé que du côté de la quantité et de la qualité de ce qui est produit: à nouveau on peut distinguer le contexte où l'incertitude est grande parce qu'on ne dispose d'information ni sur la quantité ni sur la 
probabilité d'occurrence de ce qui est échangé ou produit dans une transaction, où le contexte contraire, où l'incertitude est faible.

- le degré de mesurabilité des actifs échangés et des résultats de l'échange peut lui aussi être élevé ou faible suivant qu'on dispose ou non d'unités de mesures et de quantités.

Dans un contrat où la partie gouvernementale se limite à fournir le financement en échange de services fournis par la partie universitaire, le succès ou l'échec des transactions dépend du degré de spécificité des actifs fournis par les chercheurs universitaires, de l'incertitude des résultats de leurs recherches et de la mesurabilité des effets des résultats des recherches universitaires .

Ainsi on dira que les transactions qui sont les plus susceptibles d'échouer impliquent des actifs idiosyncratiques, un degré élevé d'incertitude et un faible degré de mesurabilité. Or plus les transactions sont susceptibles d'échouer, plus le gouvernement sera incité à introduire ou à renégocier les clauses de garantie et les clauses de monitoring susceptibles de réduire les échecs du contrat social de la recherche. Il importe donc maintenant de considérer d'une façon plus concrète les changements dans les traits des transactions qui ont amené la renégociation du contrat social de la recherche.

\section{Les changements qui ont bouleversé le contrat social de la recherche}

L'ébauche d'un contrat s'appuie sur une théorie de l'action, c'est-à-dire un énoncé des relations de causalité qui lient des actions à des effets, notamment les clauses d'un contrat avec les résultats qui en découlent. Or à ce chapitre, l'évaluation que les gouvernements ont fait au sujet des résultats de la recherche universitaire les ont amenés à réviser graduellement la plupart des clauses qui les liaient aux chercheurs universitaires. J'examinerai cette question en remontant à la création du Conseil national de la recherche du Canada (CNRC) en 1917.

\section{Changements du côté gouvernemental}

La création du CNRC a été justifiée par des arguments de déficiences dans le fonctionnement du marché privé, plus exactement, sur des observations et des perceptions voulant que l'industrie canadienne ne réalisait pas suffisamment de $\mathrm{R}$ $\& \mathrm{D}$ dans un monde où la $\mathrm{R} \& \mathrm{D}$ était en train de devenir un outil à la base de la croissance de l'industrie canadienne. Le gouvernement canadien estima alors que l'intérêt public exigeait que les fonds publics servent à compenser cette déficience du marché privé qu'était le sous-investissement dans la $R \& D$ industrielle.

Quel instrument le gouvernement canadien a-t-il développé pour compenser cette déficience de marché? Il a créé le CNRC en lui donnant le mandat de réaliser de la recherche dans ses propres laboratoires ainsi que 
d'octroyer des bourses aux étudiants et des subventions de recherche aux professeurs des universités canadiennes en se basant sur une théorie de l'action qu'on pourrait articuler dans les termes suivants: les subventions de recherche aux universités engendrent des connaissances nouvelles qui sont spontanément transformées par l'industrie en nouveaux produits et en nouveaux procédés qui entraînent une augmentation de la productivité et de la richesse (Landry, 1989).

Les travaux de Bruce Doern (1972) ont montré, qu'au fil du temps, les objectifs du CNRC se sont déplacés de l'aide à la recherche utile pour l'industrie vers l'appui à la recherche fondamentale tant du côté de ses programmes de subventions à la recherche universitaire que dans le cadre des activités de ses propres laboratoires. Ce diagnostic a été repris par maints intervenants dans le débat sur le financement de la recherche mais, notamment, et avec une force particulière, dans le volume I du Rapport Lamontagne (1971).

Paradoxe suprême, le diagnostic posé par la Commission Glassco (1969) et le comité sénatorial sur la politique scientifique canadienne indique qu'au cours des années 1960, le Canada se trouve encore dans la même situation de déficience de marché qui, cette fois, se paire à une déficience institutionnelle engendrée par un déplacement d'objectifs des arrangements institutionnels implantés pour compenser l'insuffisance de R \& D dans l'industrie. Dans son bilan, le rapport Lamontagne soutient même que le financement public de la recherche a engendré une situation de déséquilibre où le Canada investirait trop de ressources en recherche dans les universités et les laboratoires publics compte tenu des ressources consacrées à la $\mathrm{R} \& \mathrm{D}$ industriels.

Confronté à ce double constat d'échec, le gouvernement canadien est amené, vers la fin des années 1960, à réviser sa théorie de l'action pour reconnaître l'importance décisive de la demande exprimée par l'industrie. Suivant cette théorie révisée, l'innovation de produits et de procédés résulte donc de résultats de recherches en demande dans l'industrie.

Le gouvernement canadien a alors tenté de compenser ces déficiences de marché et ces déficiences institutionnelles au moyen de trois catégories d'instruments d'intervention: d'abord, en se dotant d'importants programmes d'aide à la $\mathrm{R} \& \mathrm{D}$ qui subventionnent directement les entreprises manufacturières; ensuite, en incitant les laboratoires du CNRC à mettre beaucoup plus d'accent que dans le passé sur le transfert de connaissances vers l'industrie; puis, finalement, en commençant à inciter les organismes de financement de la recherche universitaire à se doter de programmes réservés à des projets portant sur des thèmes d'importance stratégique pour l'avenir de l'industrie et de la société canadienne. 
Au cours des années 1980 et 1990 les experts posent à nouveau un diagnostic de déficience de marché et de déficience institutionnelle, mettant toutefois beaucoup plus l'accent que dans le passé sur le manque de liens et le manque de coordination entres les institutions existantes. Ce diagnostic découle cette fois d'une théorie de l'action qui met l'accent, non plus sur un type d'institutions, mais sur le réseau des institutions, ainsi que sur les liens d'interaction qui lient les institutions dans un système national d'innovation (Mowery, 1992; Nelson, 1993; Niosi, Bellon, Saviotti, \& Crow, 1992; Rosenberg, 1991; Sharp \& Pavitt, 1993). Suivant cette théorie de l'action, les variations de résultats qu'on observe d'un pays à l'autre s'expliquent moins en termes de déficiences de marché que de déficiences institutionnelles, particulièrement de déficiences du côté de la coordination formelle et informelle entre les institutions qui financent la $\mathrm{R} \& \mathrm{D}$, celles qui l'exécutent et celles qui l'utilisent pour la transformer en innovations institutionnelles et en innovations technologiques.

Le constat de déficiences de marché et de déficiences institutionnelles est donc actuellement réinterprété à la lumière de la théorie des systèmes nationaux d'innovation, non plus pour atténuer les symptômes des déficiences du marché ou des institutions, mais pour changer, adapter, voire créer de nouvelles institutions en mettant l'accent sur des clauses de coordination, des clauses de garantie et des clauses de surveillance de l'arrimage des institutions.

Cette nouvelle théorie de l'action va amener le gouvernement canadien ainsi que ceux des provinces à revoir leurs programmes de financement de la recherche en mettant l'accent sur les liens inter-institutionnels. À cet égard, on peut observer quatre tendances principales:

- les laboratoires du CNRC seront réorganisés en laboratoires plus spécialisés axés sur le transfert de technologies aux entreprises et le développement de liens avec des partenaires industriels.

- les organismes qui subventionnent la recherche universitaire seront incités à développer des programmes qui garantissent l'établissement de liens productifs de collaboration avec des partenaires socio-économiques, principalement avec l'industrie. C'est ainsi qu'émergeront de nombreux programmes de financement réservés spécifiquement à des projets de partenariats entre l'université et l'industrie.

- également, plutôt que de canaliser la quasi-totalité des fonds alloués à la recherche universitaire à travers les organismes subventionnaires que sont le CRSNG, le CRM et le CRSH, le gouvernement canadien a lancé plusieurs programmes ad hoc axés 
sur l'instauration de partenariats avec l'industrie. Les programmes de centres d'excellence, de réseaux de centres d'excellence ainsi que le programme éco-recherche constituent quelques illustrations de ces instruments que les gouvernements développent de façon à inciter les chercheurs universitaires à tisser des liens plus serrés avec des partenaires privés et publics.

- finalement, le gouvernement canadien et ceux des provinces ont contribué financièrement, à la fois par des subventions directes et des crédits d'impôts à la $\mathrm{R} \& \mathrm{D}$, à la création d'un grand nombre d'organisations de transfert de technologies. Parfois publiques, parfois privées, très souvent mixtes, ces organisations ont pour but principal de transférer la technologie vers l'industrie, de tisser des réseaux entre partenaires de recherches et partenaires industriels. Également, à la différence de la période antérieure, on retrouve un nombre de plus en plus élevé d'organisations dont le mandat est de développer des initiatives locales de développement et de transfert de la technologie.

Bref, ces changements indiquent qu'aux yeux des gouvernements la recherche universitaire s'insère dans le réseau des institutions du système canadien d'innovation où l'incitation à la coordination est souvent conditionnelle au développement de partenariats et à l'appariement de fonds.

\section{Changements du côté de la recherche universitaire}

Si les instruments d'intervention des gouvernements ont changé, il importe aussi de souligner que la recherche universitaire s'est elle aussi considérablement transformée tant du côté de la complexité, de la main d'oeuvre que des coûts. Examinons tour à tour ces facteurs.

La population des chercheurs s'est accrue très rapidement. Ainsi, le nombre de diplômés de Ph.D. en sciences et génie octroyé par les universités canadiennes est passé de 0 en 1910 à une vingtaine en 1930, à 177 en 1960 pour atteindre 1236 en 1990 (Kavanagh, 1993). L'augmentation de la population des chercheurs a été toute aussi rapide dans les autres grandes familles de disciplines. En outre, cette augmentation a été amplifiée par les gestionnaires des universités qui ont adopté diverses mesures susceptibles d'inciter une plus grande proportion de leurs professeurs à faire des demandes de subventions. $\mathrm{Au}$ total, l'augmentation du nombre de demandes de subventions ainsi que la croissance des ressources demandées se sont avérées plus élevées que la croissance des budgets des organismes subventionnaires, engendrant ainsi une diminution progressive des taux de succès et des taux de financement. 
La diminution progressive des taux de financement des projets de recherche se paire à l'augmentation de l'indice des prix de la recherche; l'apparition de normes de plus en plus strictes concernant la sécurité, le bien-être des sujets expérimentaux et les salaires du personnel de recherche se sont conjugées à l'avancement des connaissances et à une compétition de plus en plus intense pour forcer les chercheurs à utiliser des équipements de plus en plus dispendieux dans un monde où le coût de déplacements vers les sites de recherche et les colloques se sont également accrus eux aussi très considérablement. Bien que Statistique Canada ne compile pas de données sur l'indice des prix de la recherche, on peut penser qu'il s'accroît plus rapidement que l'indice des prix des biens de consommation. Nous nous retrouvons donc avec un nombre croissant de chercheurs voulant faire financer des projets qui coûtent chacun de plus en plus cher à réaliser.

Parallèlement à ces changements, l'avancement des connaissances et la compétition de plus en plus vive pour l'obtention des fonds, ont contribué à une spécialisation de plus en plus grande des chercheurs. Si cette spécialisation croissante constitue un gage de succès dans le monde de la science, elle s'avère moins efficace lorsqu'il s'agit de contribuer à la résolution de problèmes, puisque les frontières des problèmes ne coïncident à peu près jamais aux frontières des spécialités des disciplines scientifiques. À l'instar des autres intervenants dans le système national d'innovation, les chercheurs universitaires commencent à réaliser que la résolution de problèmes et le transfert de connaissances ne procèdent pas d'une logique linéaire où l'objectivité de la science et l'efficience des mécanismes du marché font passer progressivement les inventions de façon successive, d'abord, à travers un filtre technique qui sélectionne les solutions techniques les plus efficientes, ensuite, un filtre économique qui élimine les solutions scientifiques qui ne sont pas économiquement viables et, finalement, un filtre d'ajustement basé sur les mécanismes de marché qui sélectionne la meilleure innovation. Les chercheurs en sociologie de la technologie ont abondamment documenté les facteurs de résistance qui ont cours à chacune de ces étapes pour ainsi transformer cette logique linéaire en une logique non linéaire. Ce nouveau contexte a incité les chercheurs à forger des alliances et à développer des programmes et des projets de recherche avec des chercheurs d'autres disciplines et de s'engager dans la réalisation de projets véritablement inter-disciplinaires.

L'apparition de ce nouveau contexte a aussi incité les chercheurs universitaires les plus actifs à utiliser les subventions des organismes subventionnaires comme des lettres de créances leur permettant d'aller chercher 
Tableau 1

Évolution du contrat social de la recherche de 1917 à aujourd'hui.

\begin{tabular}{|c|c|c|}
\hline $\begin{array}{l}\text { Variation de la clause } \\
\text { Objet dela clause }\end{array}$ & $\begin{array}{l}\text { Rôle des chercheurs } \\
\text { universitaires }\end{array}$ & Rôle du gouvernement \\
\hline $\begin{array}{l}\text { 1. Coordination stratégique: } \\
\text { objectifs et orientations }\end{array}$ & $\begin{array}{l}\text { choix par les pairs jusque } \\
\text { vers } 1970\end{array}$ & $\begin{array}{l}\text { avant 1970: aucun } \\
\text { après 1970: choix de thèmes } \\
\text { prioritaires }\end{array}$ \\
\hline $\begin{array}{l}\text { 2. Coordination organisationnelle: } \\
\begin{array}{ll}2.1 & \text { ressources apportées } \\
\text { par chaque partie } \\
2.2 \text { façon d'utiliser les } \\
\text { ressources }\end{array}\end{array}$ & $\begin{array}{l}\text { savoir faire } \\
\text { assez grande liberté }\end{array}$ & $\begin{array}{l}\text { subventions } \\
\text { faible mais en expansion }\end{array}$ \\
\hline $\begin{array}{l}\text { 3. Coordination opérationnelle: } \\
\text { délais pour produire résultats }\end{array}$ & perspective de long terme & $\begin{array}{l}\text { perspective de plus en plus } \\
\text { axe sur le court terme }\end{array}$ \\
\hline 4. Clauses de garantie & & $\begin{array}{l}\text { avant 1970: aucune } \\
\text { depuis } 1970 \text { : } \\
\text { - thèmes } \\
\text { - appuis de partenaires } \\
\text { - \$ de partenaires } \\
\text { - équipes multidisciplinaires } \\
\text { - degré d'interactions avec } \\
\text { partenaires } \\
\text { - contributions à la formation } \\
\text { - multiplication des } \\
\text { indicateurs de résultats }\end{array}$ \\
\hline 5. Clauses de monitoring & & $\begin{array}{l}\text { avant 1970: aucune } \\
\text { depuis 1970: } \\
\text { - évaluation des arrangements } \\
\text { institutionnels de gestion } \\
\text { - arrangements institutionnels } \\
\text { d'interactions avec partenaires } \\
\text { - arrangements institutionnels } \\
\text { de transfert et de diffusion } \\
\text { de résultats } \\
\text { - financement à travers des } \\
\text { programmes ad hoc plutôt } \\
\text { que par les organismes } \\
\text { subventionnaires } \\
\text { - financement à travers des } \\
\text { subventions et mesures } \\
\text { fiscales offertes à l'industrie } \\
\text { plutôt qu'aux universités. }\end{array}$ \\
\hline
\end{tabular}


du financement dans les nouveaux programmes gouvernementaux ad hoc de subventions de la recherche universitaire qui exigent généralement des stratégies inter-disciplinaires de recherche impliquant la participation de partenaires socio-économiques. Ces programmes contribueront vraisemblablement à concentrer les fonds de recherche, mais aussi et surtout à accroître le transfert plus rapide de connaissances entre les divers participants dans le système national d'innovation. De fil en aiguille, les chercheurs sont devenus des entrepreneurs, des spécialistes de montages financiers, des gérants de personnel ainsi que des experts en relations publiques.

Les bouleversements qui sont apparus tant du côté du gouvernement que des universités ont ainsi débouché sur l'implantation du contrat social de la recherche de plus en plus explicite comportant un nombre de plus en plus élevé de clauses de garantie et de surveillance. (Tableau 1)

Dans un monde où l'interprétation dominante de la réalité soutient que le transfert efficace de connaissances exige le renforcement des liens entre les différentes institutions qui participent aux processus d'innovation, il est impossible d'envisager un retour vers une forme de contrat inconditionnel comme celle qui existait avant 1960, voire même avant 1980 .

\section{Opportunités et pistes d'action}

Les changements qui sont survenus dans le contrat social de la recherche ne doivent pas être considérés dans la seule perspective de contraintes additionnelles que les gouvernements imposent aux chercheurs et aux universités. Il importe d'entrevoir et de saisir les opportunités qui sont en train d'émerger et de se préparer à tirer avantage des nouveaux modes de financement de la recherche universitaire. Dans cet esprit, chaque université canadienne et toutes les organisations qui les représentent devraient oeuvrer à promouvoir les dividendes des investissements effectués dans la recherche en utilisant les mêmes moyens que les autres groupes d'intérêt.

Les dividendes qui résultent du financement de la recherche universitaire sont très importants tant du côté de la formation de leaders scientifiques, économiques et sociaux que du côté de la résolution de problèmes techniques, économiques et sociaux. Or, ces dividendes très importants sont méconnus et sous-évalués tant par les instances gouvernementales que par l'ensemble de la population. Il devient de plus en plus impératif de faire connaître à leur juste valeur les retombées de la recherche. Ainsi, il est grand temps que les universités et les associations qui les représentent mettent l'accent sur la dizaine de milliers d'emplois hautement qualifiés créés par les subventions de recherche dont plus de $80 \%$ servent à l'engagement de main-d'oeuvre plutôt que de s'en 
remettre au constat populaire non fondé qui prétend que les universités forment des chômeurs instruits. Également, il convient de mettre en évidence, auprès de la population aussi bien que des politiciens, les contributions de recherche susceptibles de valoriser les dividendes issus des investissements dans la recherche universitaire. Ce genre de mise en valeur ne peut se limiter, comme c'est le plus souvent le cas aujourd'hui, à produire des hebdomadaires, des magazines ou des communiqués de presse qui annoncent avec fierté les grosses subventions que les chercheurs des universités obtiennent.

Une enquête réalisée pour le compte du Fonds FCAR sur la question des liens de collaboration des universitaires du Québec montre que les chercheurs universitaires travaillent fréquemment suivant un mode de coopération relativement intense avec diverses catégories de partenaires socio-économiques: $40 \%$ de l'ensemble des répondants, qui provenaient de toutes les disciplines scientifiques, ont réalisé, au cours des cinq dernières années, des projets conjointement avec des partenaires industriels alors que $60 \%$ des répondants ont collaboré à des projets effectués avec des partenaires institutionnels, c'est-à-dire des organismes gouvernementaux et diverses catégories de groupes d'intérêt (Godin \& Landry, 1995).

Par ailleurs, les universités devraient reconnaître un principe simple de réalité qui veut que le financement de la recherche ne dépend pas de la rationalité de la logique scientifique mais plutôt de la compétition pour l'obtention de fonds publics, dans un monde où les programmes de subventions pour la recherche universitaire sont en compétition avec les demandes de financement de projets d'autres groupes socio-économiques qui paraissent tout aussi prometteurs sous l'angle des retombées socio-économiques.

Le contexte d'austérité budgétaire des gouvernements a pour effet d'accroître l'intensité de la compétition pour les fonds publics. Les coupures de budgets que viennent de subir les trois grands organismes fédéraux incitent à conclure que les universités et leurs associations n'ont pas réussi à vendre l'utilité de la recherche.

En effet, il est grand temps que les universitaires et leurs représentants comprennent qu'il n'est plus suffisant de postuler l'utilité potentielle de la recherche mais qu'il importe désormais d'en démontrer l'utilité concrète au moyen de projets précis. Il importe de décrire et d'expliquer aux politiciens et à la population que la science contribue à la création de richesses et à l'amélioration du bien-être, mais surtout, il sera de plus en plus essentiel de démontrer que les universités ont à la fois la volonté et la capacité de créer de la 
richesse et d'améliorer le bien-être de la population en collaborant étroitement avec les autres partenaires socio-économiques.

Suivant cette perspective, il importe que les universités et leurs associations commencent à compétionner avec les autres groupes d'intérêt qui tentent d'influencer le contenu des lois et des programmes gouvernementaux de dépenses budgétaires en exerçant des pressions sur différents points du processus de fabrication et d'application des programmes gouvernementaux. Les enquêtes sur le terrain démontrant plusieurs des tactiques utilisées le plus fréquemment par les groupes d'intérêt mériteraient qu'on les adaptent pour promouvoir la cause du financement de la recherche universitaire (Miller, 1987; Lehman Scholzman \& Tierney (1986):

1. Présentations de mémoires en commissions et comités parlementaires pour mettre en évidence les dividendes de la recherche, ainsi que la volonté et la capacité des universités de les produire.

2. Contacts officiels avec les politiciens (ministres) pour présenter le point de vue des universités et des chercheurs .

3. Présentations de résultats de recherche aux politiciens et aux fonctionnaires.

4. Contacts informels avec les politiciens et les fonctionnaires lors de lunchs, de congrès ou colloques.

5. Formations de coalitions avec d'autres organisations, notamment avec des partenaires socio- économiques.

6. Actions visant à influencer la façon d'appliquer les mesures gouvernementales.

7. Conférences de presse sur les "success stories" de la recherche.

8. Organisations de campagnes d'envois de lettres, de fax ou de messages sur Internet auprès des politiciens.

9. Actions visant à influencer l'agenda gouvernemental en attirant l'attention sur certains problèmes négligés nécessitant des investissements en recherche.

10. Participation à la rédaction de projets de lois, de règlements ou de programmes gouvernementaux ayant des implications pour la recherche universitaire.

11. Participation à des conseils consultatifs et à des groupes de travail ayant des incidences sur la recherche universitaire. 
12. Actions visant à alerter les députés des conséquences fâcheuses des projets gouvernementaux concernant la recherche universitaire.

13. Actions visant à alerter les fonctionnaires du Conseil du Trésor et des ministères de tutelle des effets négatifs de projets gouvernementaux concernant les organismes subventionnaires.

14. Publications d'annonces dans les journaux visant à influencer l'agenda et les projets des gouvernements en matière de recherche.

15. Appuis à des candidat(e)s locaux favorables à la défense et à la promotion du financement de la recherche universitaire .

Il me paraît impératif que chaque université apprivoise ces tactiques de façon à y recourir de plus en plus souvent, de plus en plus régulièrement, mais surtout, de plus en plus efficacement. L'avenir du financement de la recherche universitaire et du contrat social qui lie les universités au gouvernement en dépend.

\section{Conclusion}

Le contrat social de la recherche s'est progressivement modifié de façon à incorporer un nombre de plus en plus élevé de clauses de garantie et de clauses de monitoring au fur et à mesure que la théorie de l'action qui inspire le développement des interventions gouvernementales en est venue à mettre l'accent, non plus seulement sur l'offre ou sur la demande de connaissances, mais sur les liens d'interdépendance qui arriment les différentes institutions qui participent au développement, aux transferts et à l'utilisation des connaissances. Cette évolution dans la compréhension des relations de causalité qui existent entre la science, l'économie et les institutions a progressivement mais profondément bouleversé tant les modes de financement que les façons de faire de la science.

Dans ce contexte où la science devient un facteur de production comme les autres, les universités devront de plus en plus investir de ressources dans la valorisation de leurs activités et résultats de recherche en utilisant, à l'instar des autres groupes d'intérêt, les tactiques les plus susceptibles d'influencer le maintien ou l'augmentation des ressources que les gouvernements allouent à la recherche universitaire. 


\section{References}

Brousseau, E. (1993). L'économie des contrats. Paris: Presses Universitaires de France.

Bryson, J.M., \& Smith Ring, P. (1990). A transaction based approach to policy intervention. Policy Sciences, 23(3), 205-229.

Doern, G.B. (1972). Science and politics in Canada. Montréal: McGill-Queen's University Press.

Glassco. (1969). Commission royale d'enquête sur l'organisation du gouvernement. Ottawa: Imprimeur de la reine.

Godin, B., \& Landry, R. (1995). L'avenir de la collaboration scientifique au Québec: une analyse basée sur la convergence d'indicateurs. Québec: Rapport préparé pour le Fonds FCAR.

Guston, D.H., \& Keniston, K. (Eds.). (1994). The fragile contract: University science and the federal government. Cambridge: The MIT Press.

Kavanagh. R.J. (1993). Educational and scientific infrastructure. In P. Dufour \& J. De la Mothe (eds.), Science and technology in Canada, p. 107. Essex: Longman.

Lamontagne, M. (1971). Une politique scientifique canadienne. 4 volumes, Ottawa: Imprimeur de la reine (aussi connu sous le nom de rapport du comité sénatorial sur la politique scientifique).

Landry, R. (1989). Barriers to efficient monitoring of science, technology and innovation through public policy. Science and Public Policy, 16(6), 345-352.

Lehman Schlozman, K., \& Teirney, J.T. (1986). Organized interest and American democracy. New York: Harper and Row Publishers.

Miller, C. (1987). Lobbying government. Oxford, Basil Blackwell.

Mowery, D.C. (1992). The U.S. national innovation system: Origins and prospects for change. Research Policy, 21,125-144.

Nelson, R.R. (1993). National innovation systems: A comparative analysis. Oxford, Oxford University Press.

Niosi, J., Bellon, B., Saviotti, P., \& Crow, M. (1992). Les systèmes nationaux d'innovation: à la recherche d'un concept utilisable. Problèmes économiques, no 2 311, février 1993, 22.

Rosenberg, N. (1991). Critical issues in science policy research. Science and Public Policy, 18(6), 335-346.

Sharp, M., \& Pavitt, K. (1993). Technology policy in the 1990's: Old trends and new realities, Journal of Common Market Studies, 31(2), 129-151.

Williamson, O.E. (1985). The economic institutions of capitalism. New York: The Free Press. 